\title{
miR-130b promotes bladder cancer cell proliferation, migration and invasion by targeting VGLL4
}

\author{
XIAOWU LIU ${ }^{1,2}$, CHUIZE KONG ${ }^{1,2}$ and ZHE ZHANG ${ }^{1,2}$ \\ ${ }^{1}$ Department of Urology and ${ }^{2}$ Institute of Urology, The First Hospital of China Medical University, \\ Shenyang, Liaoning 110001, P.R. China
}

Received March 27, 2017; Accepted March 1, 2018

DOI: $10.3892 /$ or.2018.6300

\begin{abstract}
Bladder cancer (BCa) is the most common urological cancer, and more and more evidence suggests that microRNAs (miRNAs) play an important role in BCa pathogenesis. Aberrant miR-130b expression has been reported in several types of cancers. The aim of the present study was to elucidate the effects of miR-130b on BCa progression. miR-130b expression in BCa cell lines and tissues was detected using real-time PCR (RT-PCR), and vestigial-like protein 4 (VGLL4) expression in tissue specimens and $\mathrm{BCa}$ cells that had been transfected with miR-130b mimics and inhibitors was detected using western blotting. Dual-luciferase reporter assay was performed to confirm whether the VGLL4 gene is a direct target of miR-130b, and in vitro cell function testing, Cell Counting Kit-8 (CCK-8) assay, colony formation assay, wound healing and Transwell assays were performed to examine $\mathrm{BCa}$ cell proliferation, migration and invasion ability after the cells were transfected with miR-130b mimics and inhibitors and VGLL4 siRNA. miR-130b was found to be upregulated in $\mathrm{BCa}$ cells and tissues. miR-130b overexpression promoted $\mathrm{BCa}$ cell proliferation, migration and invasion, whereas miR-130b inhibition had the opposite effects. Dualluciferase reporter assay confirmed that the VGLL4 gene was a direct target of miR-130b and that VGLL4 suppression was crucial for miR-130b-induced BCa cell proliferation, migration and invasion. The present study showed that miR-130b was significantly upregulated in $\mathrm{BCa}$ and may play an oncogenic role in $\mathrm{BCa}$ occurrence and development by targeting VGLL4. miR-130b may be a potential therapeutic target in the treatment of $\mathrm{BCa}$.
\end{abstract}

Correspondence to: Professor Chuize Kong, Department of Urology, Institute of Urology, The First Hospital of China Medical University, 155 North Nanjing Street, Heping, Shenyang, Liaoning 110001, P.R. China

E-mail: kongchuize_cmu@sina.com

Key words: bladder cancer, miRNA, miR-130b, VGLL4, proliferation, migration and invasion

\section{Introduction}

Bladder cancer (BCa) is the most common malignant tumor in the urinary system, and more than 300,000 new cases of the disease are diagnosed each year worldwide $(1,2)$. BCa can be divided into the following two major types: non-muscleinvasive bladder cancer (NMIBC) and muscle-invasive bladder cancer (MIBC). Patients with NMIBC have a good prognosis, and the 5-year survival rate for such patients is over $88 \%$ (3); however, $\sim 70 \%$ of affected patients will experience disease recurrence at the same position or another bladder site (4). The 5 -year survival rate for patients with MIBC is only $~ 60 \%(3,5)$. Hence, studies investigating the potential mechanisms underlying $\mathrm{BCa}$ occurrence and development are urgently needed. Recently, the results of an increasing number of studies have suggested that microRNAs (miRNAs) play an important role in BCa pathogenesis, and may thus provide clinicians with new therapeutic targets and strategies for treating the disease (6-9).

miRNAs are a class of conserved small non-coding RNAs that exert their effects by binding to the 3 ' untranslated regions (3'UTRs) of their target messenger RNAs (mRNAs), leading to mRNA degradation or translational inhibition and, ultimately, gene expression regulation (10). The results of previous studies indicate that miRNAs play important roles in cancer occurrence and development as oncogenes or tumor-suppressor genes (11). miRNAs can affect cell proliferation, apoptosis, invasion, metastasis and epithelial-mesenchymal transition (EMT) (12-14). miR-130b, which is located at the 22q11 locus (15), has been shown to be aberrantly expressed in some cancers, as it is overexpressed in renal cell (16) and hepatocellular carcinoma (17), and glioma (18), but is downregulated in papillary thyroid (19) and endometrial carcinoma (20), and prostate cancer (21).

In a preliminary experiment, we found that miR- $130 \mathrm{~b}$ was upregulated in BCa cells and tissues and that ectopic miR-130b expression was closely related to $\mathrm{BCa}$ cell proliferation, migration and invasion, indicating that miR-130b may function in BCa progression.

Using bioinformatic analysis software, we identified vestigial-like protein 4 (VGLL4) as the downstream target gene of miR-130b. VGLL proteins are newly emerging TEAD-interacting partners and transcriptional cofactors that participate in tumorigenesis. Unlike other members of the VGLL family, VGLL4 contains an extra TDU domain, which 
is thought to be functionally distinct from the other functional domains of the protein, and has been identified as a transcriptional inhibitor that inhibits YAP-induced tumor growth and development in Drosophila and humans (22). The role of VGLL4 in gastric, pancreatic and lung cancer, and esophageal squamous cell carcinoma has recently attracted more attention. VGLL4 is considered a new tumor-suppressor gene (23-28).

This study showed that the VGLL4 gene was a direct target of miR-130b and that VGLL4 downregulation mediated by miR-130b played a critical role in BCa cell proliferation, migration and invasion.

\section{Materials and methods}

Cell cultures and clinical tissue specimens. The indicated human BCa cell lines (T24, 5637 and J82) and immortalized human bladder urothelial cell line (SV-HUC-1) were obtained from the Cell Bank of the Chinese Academy of Sciences (Shanghai, China) and cultured in HyClone ${ }^{\mathrm{TM}}$ RPMI-1640 medium supplemented with $10 \%$ fetal bovine serum (FBS) (GE Healthcare Life Sciences, HyClone Laboratories, Logan, UT, USA). All cells were cultured in a humidified incubator at $37^{\circ} \mathrm{C}$ with $5 \% \mathrm{CO}_{2}$ and $95 \%$ air. From January 2016 to September 2016, thirty pairs of clinical tissue specimens (bladder tumor tissue specimens and adjacent non-tumor bladder tissue specimens), including 19 men and 11 women with a median age of 63 years, were obtained from patients undergoing transurethral bladder tumor resection (17 cases) or radical cystectomy (13 cases) at the Department of Urology, The First Hospital of China Medical University. All these cases were staged by the 2002 UICC TNM classification. Of these 30 cases of $\mathrm{BCa}, 17$ cases were NMIBC $(\leq \mathrm{pT} 1)$, and the remaining cases were MIBC (>pT1). The cases were also classified according to the WHO 2004 for grade, including 19-low grade papillary urothelial carcinoma, and 11-high grade papillary urothelial carcinoma. No patients had received radiotherapy, chemotherapy or adjuvant therapy before surgery. All specimens were obtained after each patient provided written informed consent to participate in this study, which was approved by the Research Ethics Committee of The First Hospital of China Medical University. Some specimens were fixed in formalin, while others were immediately placed in liquid nitrogen and stored at $-80^{\circ} \mathrm{C}$ for further analysis.

RNA extraction and real-time PCR (RT-PCR). To detect VGLL4 mRNA, we extracted total RNA from cultured cells and frozen tissues using Invitrogen ${ }^{\mathrm{TM}} \mathrm{TRIzol}^{\mathrm{TM}}$ reagent (Thermo Fisher Scientific, Inc., Waltham, MA, USA), according to the manufacturer's instructions. cDNA was synthesized with a PrimeScript ${ }^{\mathrm{TM}}$ RT reagent kit (Takara, Shiga, Japan), and RT-PCR was performed using a SYBR ${ }^{\circledR}$ Premix Ex Taq ${ }^{\mathrm{TM}}$ kit (Takara) in a Roche LightCycler ${ }^{\circledR} 480$ (Roche, Basel, Switzerland), according to the manufacturers' instructions. To detect miR-130b, we extracted total RNA (including microRNA) using a miRNeasy ${ }^{\mathrm{TM}}$ Mini kit (Qiagen, Hilden, Germany), according to the manufacturer's instructions. cDNA was synthesized using a miRCURY LNA ${ }^{\mathrm{TM}}$ Universal cDNA Synthesis kit II (Exiqon A/S, Vedbaek, Denmark), and RT-PCR was performed with miRCURY LNA $^{\mathrm{TM}}$ ExiLENT SYBR ${ }^{\circledR}$-Green Master Mix (Exiqon A/S), according to the manufacturer's instructions. miR-130b-3p and VGLL4 expression levels were normalized to U6 and $\beta$-actin expression levels, respectively. Relative expression levels were calculated using the $2-\Delta \mathrm{Ct}$ method. The primers for VGLL4 were: 5'-GCTGGTTTTCTTTGCTAGCCC-3' (forward) and 5'-CACCGGCAGGGTCTGTATTC-3' (reverse), and the primers for $\beta$-actin were: 5'-CTCACCATGGATGATGATAT CGC-3' (forward) and 5'-AGGAATCCTTCTGACCCATGC-3' (reverse). The hsa-miR-130b-3p and U6 LNA ${ }^{\mathrm{TM}}$ PCR primer sets were provided by Exiqon.

Transient transfection. T24 and 5637 cells were transfected with hsa-miR-130b-3p mimics, negative-control mimics, hsamiR-130b-3p inhibitors, negative-control inhibitors and VGLL4 siRNA (GenePharma, Shanghai, China) using

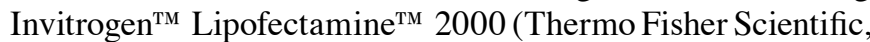
Inc.) according to the manufacturer's instructions. The VGLL4 siRNA sense sequence was as follows: 5'-UCUGAACAAGA CUGCCAAUTT-3', and the VGLL4 siRNA antisense sequence was as follows: 5'-AUUGGCAGUCUUGUUCAGATT-3'. The expression levels of all transfected genes were confirmed with RT-PCR or western blotting.

Luciferase assays. A total of 5637 cells were seeded in 24-well plates and incubated for $24 \mathrm{~h}$ before transfection. miR-130b NC/pmirGLO-VGLL4-3'UTR-WT, miR-130b mimic/pmirGLO-VGLL4-3'UTR-WT, miR130b NC/pmirGLO-VGLL4-3'UTR-MUT and miR-130b mimic/pmirGLO-VGLL4-3'UTR-MUT (synthesized by GenePharma, Shanghai, China) were transiently co-transfected into the cells using Lipofectamine 2000. Cell lysates were gathered $48 \mathrm{~h}$ after transfection and assessed using a Dual-Luciferase Reporter Assay kit (Promega, Madison, WI, USA), according to the manufacturer's protocol. All experiments were performed in triplicate.

Cell proliferation assay. Cell proliferation assay was conducted with the Cell Counting Kit-8 (CCK-8) (Dojindo, Tokyo, Japan). The cells were seeded in 96 -well plates $\left(3 \times 10^{3}\right.$ cells/well) and incubated for $24 \mathrm{~h}$ before transfection. Ten microliters of CCK-8 was added to every well at 1,2,3,4 and 5 days after transfection. Then, the cells were incubated at $37^{\circ} \mathrm{C}$ for $2 \mathrm{~h}$, after which the absorbance was measured at $450 \mathrm{~nm}$ using a microplate reader. All experiments were performed in triplicate.

Colony formation assay. The cells $\left(5 \times 10^{2}\right.$ cells/well) were seeded in 6-well plates in complete growth media and incubated for 2 weeks. The cell colonies were then fixed with methanol and stained with $0.1 \%$ crystal violet before being imaged and counted (defined as $>50$ cells/colony) using an AID iSpot Reader (Autoimmun Diagnostika GmbH, Strassberg, Germany). The experiment was performed three times for each cell line.

Wound healing assay. The cells were seeded in 6-well plates $\left(5 \times 10^{5}\right.$ cells/well) and incubated until they reached $100 \%$ confluence. Then, the cells were wounded by a $200-\mu 1$ pipette tip, and a cross-shape was generated in the monolayer. The cells were subsequently washed with PBS for cellular debris removal and cultured for $24 \mathrm{~h}$. Then, the widths of wounds 

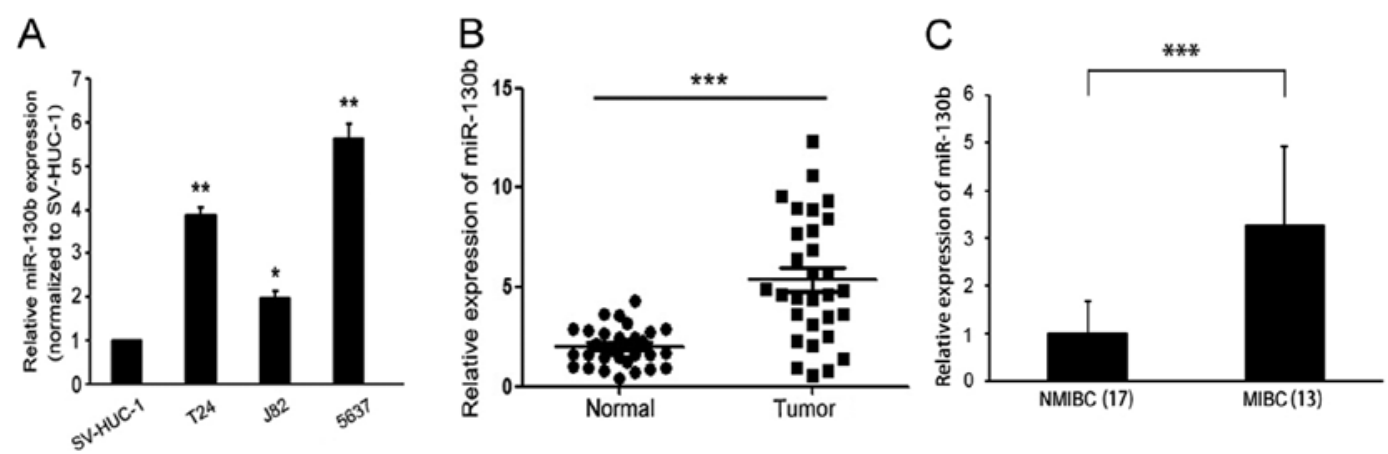

Figure 1. miR-130b expression is upregulated in bladder cancer (BCa) cell lines and tissues. (A) Analysis of miR-130b expression in BCa cells was performed with RT-PCR. miR-130b expression levels in the BCa cell lines T24, J82 and 5637 were compared with those in SV-HUC-1 cells, which served as a control. (B) miR-130b expression was examined in 30 pairs of BCa tissues and adjacent non-tumor tissues. (C) miR-130b expression in muscle-invasive bladder cancer (MIBC) (13 cases) compared with that in non-invasive bladder cancer (NMIBC) (17 cases) tissues. Data represent the mean \pm SD of three independent experiments; ${ }^{*} \mathrm{P}<0.05,{ }^{* *} \mathrm{P}<0.01,{ }^{* * *} \mathrm{P}<0.001$.

were measured under a light microscope (Olympus Corp., Tokyo, Japan). The widths between the cells which migrated the furthest on each side were measured to calculate the relative wound closure area. The experiment was performed three times for each cell line.

Invasion assay. Cell invasion assay was performed with Corning Transwell insert chambers (Corning Incorporated, New York, NY, USA) with an $8.0-\mu \mathrm{m}$ pore size. Matrigel (Corning Incorporated) was used to coat the top chamber. A total of $2 \times 10^{4}$ cells were suspended in $200 \mu \mathrm{l}$ of serum-free medium and seeded in the upper chamber, and RPMI-1640 containing $10 \%$ FBS, which was used as a chemoattractant, was added to the lower chamber. After incubating for $24 \mathrm{~h}$, the cells in the upper chamber were removed with cotton swabs, and the cells that had passed through the membrane to invade the lower chamber were fixed with methanol and stained with $0.1 \%$ crystal violet before being counted under a light microscope. All experiments were performed in triplicate.

Western blot analysis. The transfected cells and frozen tissues (including the tumor and adjacent non-tumorous tissues) were lysed, and the extracted proteins were quantified by a BCA Protein Assay kit (Pierce Biotechnology, Rockford, IL, USA). Equal quantities of protein were separated by $10 \%$ SDS-PAGE and transferred to polyvinylidene fluoride (PVDF) membranes, which were blocked with $5 \%$ non-fat milk at room temperature for $2 \mathrm{~h}$, and then incubated with the appropriate primary antibody at $4^{\circ} \mathrm{C}$ overnight. Following 3 washes in Tris-buffered saline containing $0.1 \%$ Tween-20 (TBST), the membranes were incubated with the appropriate secondary antibody for $2 \mathrm{~h}$ at $37^{\circ} \mathrm{C}$. Specific band signals were detected using a chemiluminescence system (Bio-Rad, Philadelphia, PA, USA) and analyzed using ImageJ Software (National Institutes of Health, Bethesda, MD, USA) in accordance with the manufacturer's protocol. The primary antibody rabbit polyclonal anti-VGLL4 (1:1,000; cat. no. V2890; Sigma-Aldrich; Merck KGaA, Darmstadt, Germany) and a secondary antibody to mouse $\operatorname{IgG}$ (1:5,000; cat. no. ab193651; Abcam, Cambridge, MA, USA) or rabbit IgG (1:5,000; cat. no. ab191866; Abcam) were used for the experiments. The protein levels were normalized to those of GAPDH (1:1,000; cat. no. ab8245; Abcam).
Statistical analysis. All data were processed with SPSS 13.0 (SPSS, Inc., Chicago, IL, USA) and presented as the mean \pm standard deviation $(\mathrm{SD})$ of three independent experiments. Differences were evaluated and analyzed with the Student's t-test. P-values $<0.05$ were considered statistically significant.

\section{Results}

miR-130b expression is upregulated in BCa. RT-PCR was used to detect miR-130b expression levels in BCa cell lines and tissues. miR-130b was found to be significantly elevated in the indicated BCa cell lines (T24, J82 and 5637) compared with the immortalized human bladder epithelial cell line (SV-HUC-1) (Fig. 1A). We also examined miR-130b expression levels in $30 \mathrm{BCa}$ clinical tissue samples and matched adjacent non-tumor tissue samples. The results of the analysis showed that miR-130b expression levels in BCa tissues were significantly higher than those in matched adjacent non-tumor tissues (Fig. 1B). In addition, miR-130b expression levels in the MIBC tissues were significantly higher than those in the NMIBC tissues (Fig. 1C). These results indicated that miR-130b expression levels were upregulated in $\mathrm{BCa}$, suggesting that miR-130b may play a role in promoting tumor progression in $\mathrm{BCa}$.

miR-130b promotes cancer cell proliferation, migration and invasion. To further investigate the potential role of $\mathrm{miR}-130 \mathrm{~b}$ in the promotion of tumor progression in $\mathrm{BCa}$, we transfected miR-130b-3p mimics and miR-130b-3p mimic controls (as control) into the T24 and 5637 cells and confirmed miR-130b-3p overexpression by RT-PCR (data not shown). We used CCK- 8 and colony formation assays to assess the effects of miR-130b on cell proliferation. The results of the CCK- 8 assay indicated that miR-130b overexpression significantly increased $\mathrm{BCa}$ cell growth rates compared with the negative control (NC) cell growth rates (Fig. 2A). The results of the colony formation assay showed that $\mathrm{BCa}$ cells overexpressing miR-130b formed a higher number of colonies than the control NC cells (Fig. 2B). Additionally, the results of the wound healing assay showed that the migration ability of BCa cells overexpressing miR-130b was significantly higher than that 
A

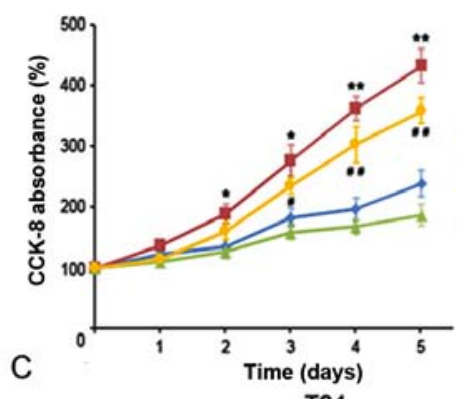

T24
B

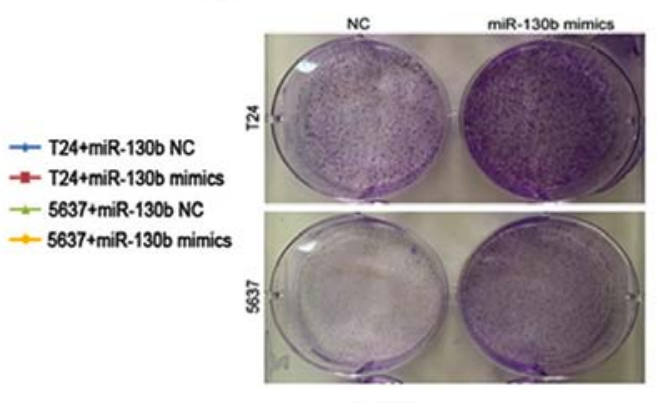

5637

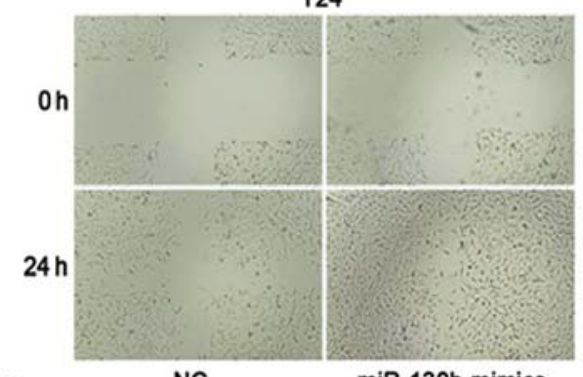

D NC miR-130b mimics NC

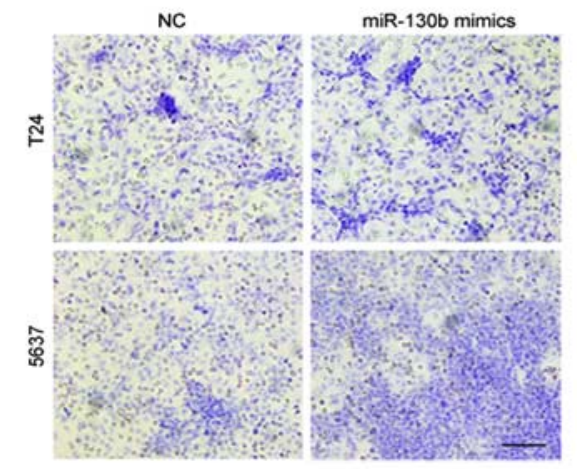

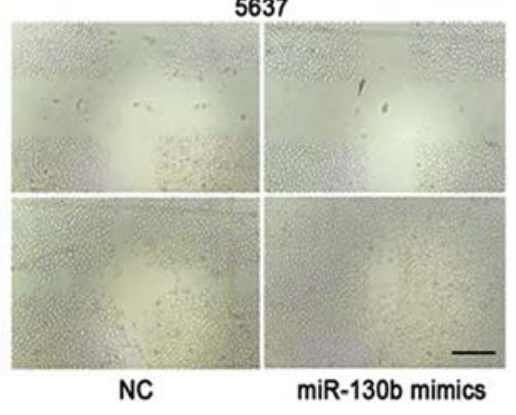

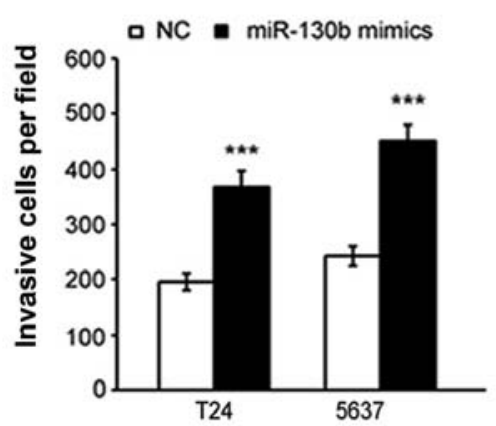

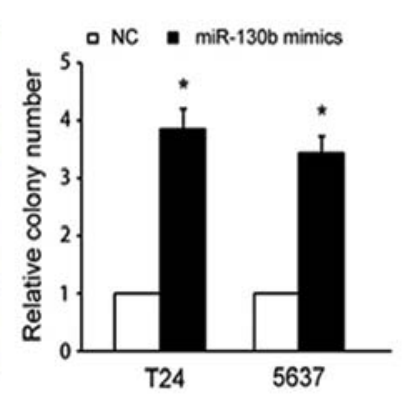

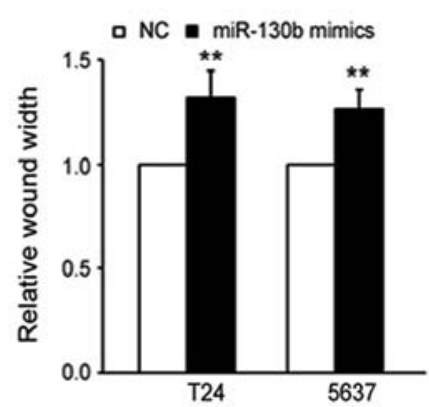

Figure 2. miR-130b promotes bladder cancer (BCa) cell proliferation, migration and invasion. (A) CCK-8 assay was performed to detect the cell proliferation ability of T24 and 5637 cells transfected with miR-130b-3p mimics and miR-130b-3p mimic controls. (B) Representative micrographs and quantification of crystal violet-staining in the colony formation assay of the indicated cells. (C) Effects on the migration ability of the indicated cells as demonstrated by wound healing assay; Scale bar, $100 \mu \mathrm{m}$. (D) Representative results of the invasion assay of the indicated cells; Scale bar, $100 \mu \mathrm{m}$. Data represent the mean \pm SD of three independent experiments; ${ }^{*} \mathrm{P}<0.05,{ }^{* *} \mathrm{P}<0.01,{ }^{* * *} \mathrm{P}<0.001,{ }^{*} \mathrm{P}<0.05,{ }^{\# \#} \mathrm{P}<0.01$ compared with the corresponding $\mathrm{NC}$ group.

of the control NC cells (Fig. 2C). Furthermore, the results of the Transwell assay indicated that miR-130b overexpression caused significant increases in the invasive capabilities of $\mathrm{BCa}$ cells compared with that of the control NC cells (Fig. 2D). All of these results indicated that miR-130b overexpression promoted $\mathrm{BCa}$ cell proliferation, migration and invasion.

miR-130b inhibition suppresses BCa proliferation, migration and invasion. To confirm the role of $\mathrm{miR}-130 \mathrm{~b}$ in $\mathrm{BCa}$ proliferation, migration and invasion, we performed loss-offunction studies by transfecting miR-130b-3p inhibitors and miR-130b-3p inhibitor controls [as control (INC)] into T24 and 5637 cells. The results of the CCK- 8 and colony formation assays showed that miR-130b suppression significantly decreased the proliferative capacity of the $\mathrm{BCa}$ cells compared with that of the INC cells (Fig. 3A and B). Additionally, the results of the wound healing and Transwell assays indicated that miR-130b inhibition caused a significant decrease in the migratory and invasive capabilities of the $\mathrm{BCa}$ cells compared to those of the INC cells (Fig. 3C and D). These results indicated that miR-130b knockdown inhibited BCa cell proliferation, migration and invasion.

VGLL4 is a direct target gene of miR-130b in BCa cells. To further explore the molecular mechanisms underlying the effects of miR-130b in BCa, we used bioinformatic analysis software (TargetScan, miRBase) to predict the target genes of miR-130b. VGLL4 was identified as one of the potential target genes of miR-130b in BCa cells (Fig. 4F). Regarding the clinical specimens, VGLL4 protein expression levels in BCa tissues were significantly decreased compared with those in the adjacent non-tumor bladder tissues, and VGLL4 protein expression levels were lower in MIBC tissues than in NMIBC tissues (Fig. 4C). In BCa cells, VGLL4 protein expression levels were decreased in the miR-130b-overexpressing cells, while VGLL4 protein expression levels were significantly increased in the miR-130b-silenced cells (Fig. 4A and B). However, there was no significant difference in VGLL4 mRNA expression levels between the two cell types (data not shown). miR-130b expression levels were negatively correlated 
A

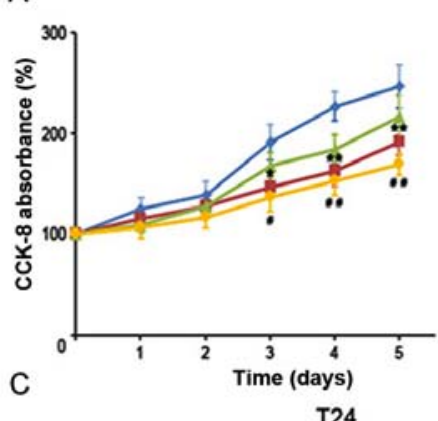

24

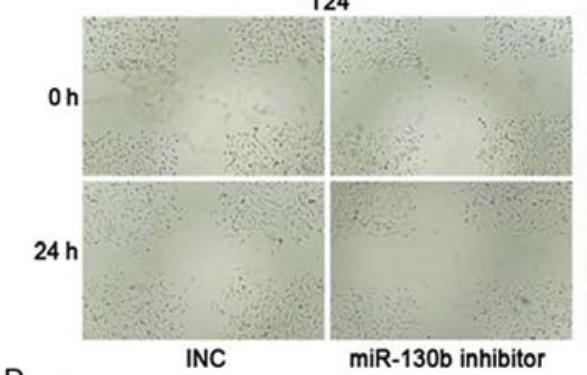

D

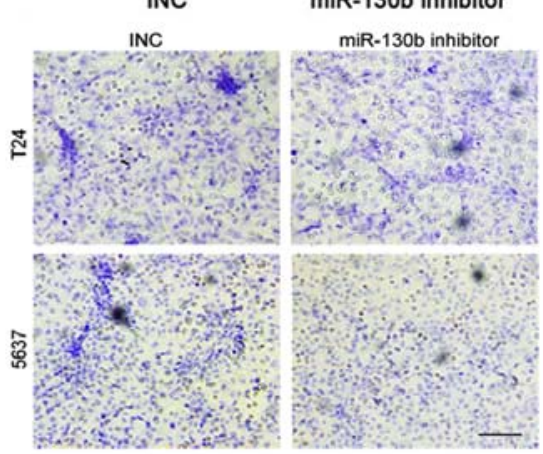

B

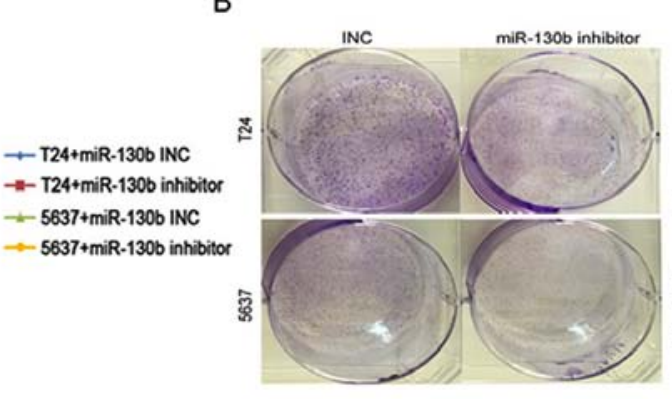

5637
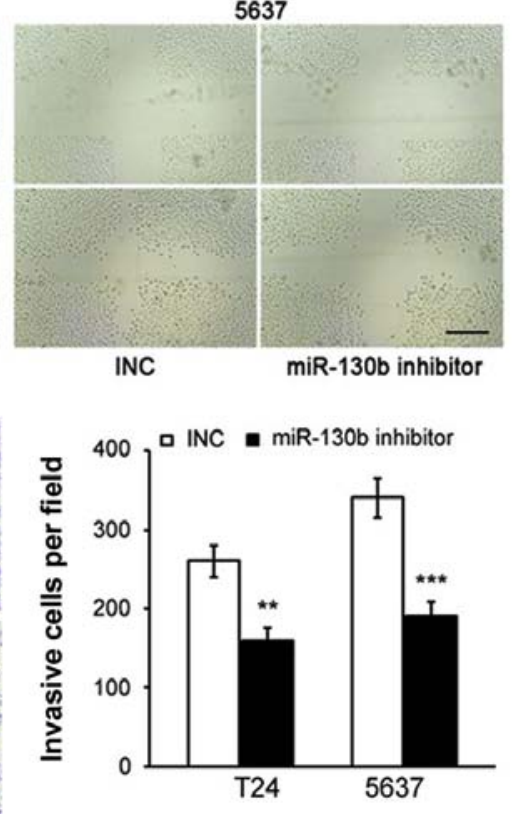
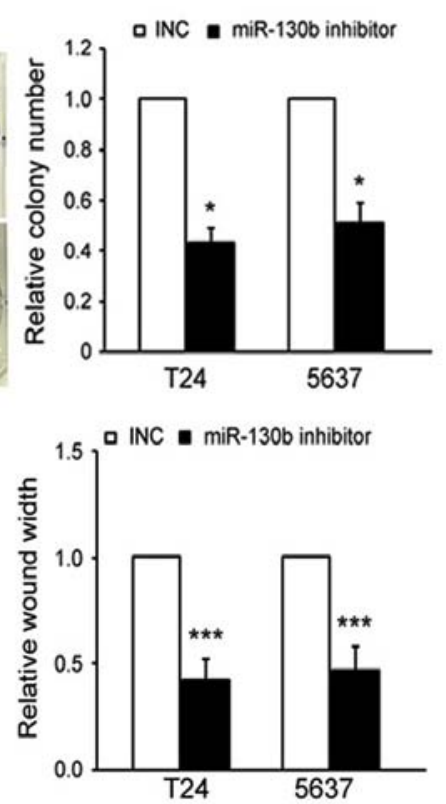

Figure 3. miR-130b inhibition suppresses bladder cancer (BCa) cell proliferation, migration and invasion. (A) CCK-8 assay was performed to detect the cell proliferation ability of T24 and 5637 cells transfected with the miR-130b-3p inhibitor and miR-130b-3p inhibitor control (INC). (B) Representative micrographs and quantification of crystal violet staining in the colony formation assay of the indicated cells. (C) Effects on the migration ability of the indicated cells, as demonstrated by wound healing assay; Scale bar, $100 \mu \mathrm{m}$. (D) Representative results of the invasion assay of the indicated cells; Scale bar, $100 \mu \mathrm{m}$. Data represent the mean $\pm \mathrm{SD}$ of three independent experiments; ${ }^{*} \mathrm{P}<0.05,{ }^{* *} \mathrm{P}<0.01,{ }^{* * *} \mathrm{P}<0.001,{ }^{\#} \mathrm{P}<0.05,{ }^{\# \#} \mathrm{P}<0.01$ compared with the corresponding INC group.

with those of VGLL4 in the BCa tissue specimens ( $r=-0.7757$, $\mathrm{P}<0.0001$ ) (Fig. 4D). To determine whether VGLL4 is regulated by the direct binding of miR-130b to its 3'UTR, we performed luciferase assays. PmirGLO-VGLL4-3'UTR-WT and miR-130b mimics were co-transfected into 5637 cells and caused a significant decrease in luciferase activity in those cells compared with control cells. In contrast, pmirGLO-VGLL4-3'UTR-MUT and miR-130b mimics were co-transfected into 5637 cells and caused no significant changes in luciferase activity in the treated group compared with the NC group (Fig. 4E). These results suggested that the VGLL4 gene was a direct target of miR-130b in BCa cells.

VGLL4 suppression is crucial for miR-130b-induced BCa cell proliferation, migration and invasion. To determine whether miR-130b-mediated $\mathrm{BCa}$ cell proliferation, migration and invasion is attributable to VGLL4, we transfected a miR-130b inhibitor/VGLL4-siRNA and miR-130b inhibitor/control vector into T24 and 5637 cells (Fig. 5A). The results of the abovementioned assays showed that VGLL4 inhibition resulted in significantly increased BCa cell proliferation (Fig. 5B and C), migration (Fig. 5D) and invasion (Fig. 5E) ability in the cells transfected with the miR-130b inhibitor. These results showed that the inhibitory effects of the miR-130b inhibitor on $\mathrm{BCa}$ cell proliferation, migration and invasion were significantly reversed by VGLL4 suppression. Hence, we confirmed that miR-130b-induced cell proliferation, migration and invasion were facilitated by VGLL4 suppression.

\section{Discussion}

More and more studies have shown that aberrant miRNA expression plays an oncogenic or tumor suppressive role. The oncogenic effects are exerted by tumor suppressor gene repression, and the tumor suppressive effects are exerted by oncogene inhibition $(29,30)$. The important roles of miRNAs in cancer occurrence and development have also been experimentally demonstrated in animal models (31). Bladder cancer (BCa) is the most common malignant tumor in the urinary system, hence, increasing numbers of studies have focused on $\mathrm{BCa}$ biological characteristics, occurrence and development and have elucidated the roles of miRNAs in BCa. For example, a study reported that $\mathrm{miR}-200 \mathrm{c}$ functions as an oncogene in $\mathrm{BCa}$ and that miR-200c downregulation significantly inhibits 


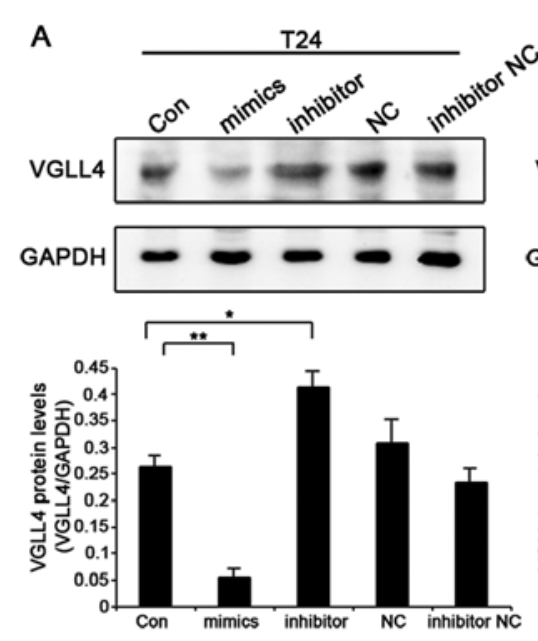

D

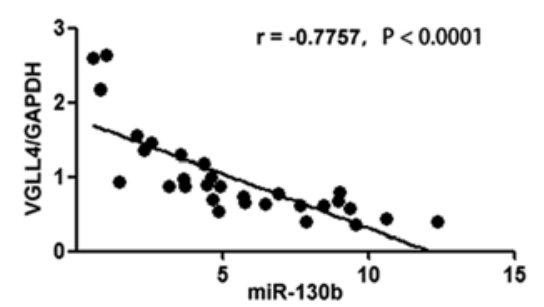

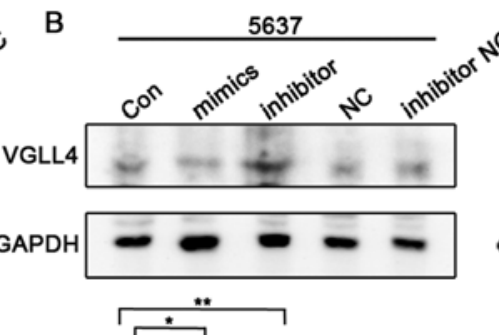

C C
VGLL4

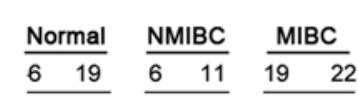
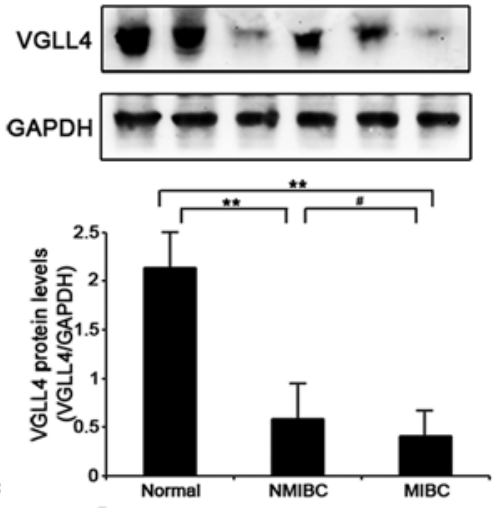

E

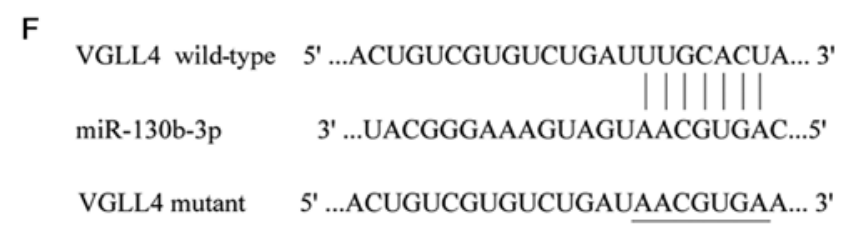

Figure 4. miR-130b directly targets VGLL4 in bladder cancer (BCa) cells. (A) VGLL4 protein expression levels in T24 cells transfected with miR-130b mimics and inhibitors compared with those in the negative control (NC) cells, as demonstrated by western blotting. (B) VGLL4 protein expression levels in 5637 cells transfected with miR-130b mimics and inhibitors compared with those in the NC cells, as analyzed by western blotting. (C) Western blot analysis of VGLL4 expression in BCa tissues, non-muscle-invasive (NMIBC) tissues (T6, T11) and muscle-invasive (MIBC) tissues (T19, T22) compared with those in adjacent non-tumor bladder tissues (N6, N19). (D) Correlation between miR-130b and VGLL4 expression in BCa tissues. (E) Luciferase activity levels were measured by luciferase assay in the indicated cells. (F) Putative sequence of miR-130b, with binding sites in the VGLL4 3'UTR. Data represent the mean \pm SD of three independent experiments; ${ }^{*} \mathrm{P}<0.05,{ }^{* *} \mathrm{P}<0.01,{ }^{*} \mathrm{P}<0.05$. UTR, untranslated region.

BCa cell migration and invasion (32). Another study reported that miRNA-206 acted as a tumor suppressor in BCa by targeting YRDC; inhibiting BCa cell proliferation, colony formation, migration and invasion; and inducing cell cycle arrest at G0/G1 phase (33). Moreover, the expression levels of some miRNAs, including miR-125b, miR-30b, miR-204, miR-99a and miR-532-3p, were significantly downregulated in patient urine supernatants. These miRNAs could be used as promising diagnostic markers for the non-invasive diagnosis of BCa (34).

In the present study, the role of miR-130b in BCa was studied in further detail. The results of this study showed that miR-130b expression levels in surgically resected tumor specimens were significantly higher than those in adjacent non-tumor bladder tissue specimens. Moreover, higher miR$130 \mathrm{~b}$ expression levels were observed in MIBC tissues than in NMIBC tissues. We also studied the effects of miR-130b gain/loss-of-function on various aspects of T24 and 5637 cell behaviors. We found that miR-130b overexpression or knockdown promoted or inhibited $\mathrm{BCa}$ cell proliferation, migration and invasion. Overall, we concluded that miR-130b may be an oncogene and plays an important role in the pathogenesis of BCa.

To study the detailed mechanisms underlying the function of miR-130b in $\mathrm{BCa}$, we identified the downstream target gene of miR-130b using bioinformatics analysis software (miRBase, TargetScan). Bioinformatics analysis demonstrated that VGLL4 may be a direct target of miR-130b in BCa. VGLL4 is a member of the VGLL family, and VGLL proteins have recently emerged as a new TEAD-interacting partners and participate in cancer development. VGLL proteins are transcriptional cofactors. Recently, more attention has been devoted to the role of VGLL4 in cancer. VGLL4 was believed to act as a tumor suppressor gene in many types of cancer (24-26,35). The results of a previous study indicated that VGLL4 directly competes with YAP to bind TEADs and that VGLL4-mimicking peptides potently suppressed gastric cancer tumor growth in vitro and in vivo (23). Previous study demonstrated that YAP was upregulated in some $\mathrm{BCa}$ cell (5637) and overexpressed YAP promoted BCa cell growth and migration (36). Furthermore, the inhibition of YAP expressions significantly increased cytotoxic drug sensitivity 

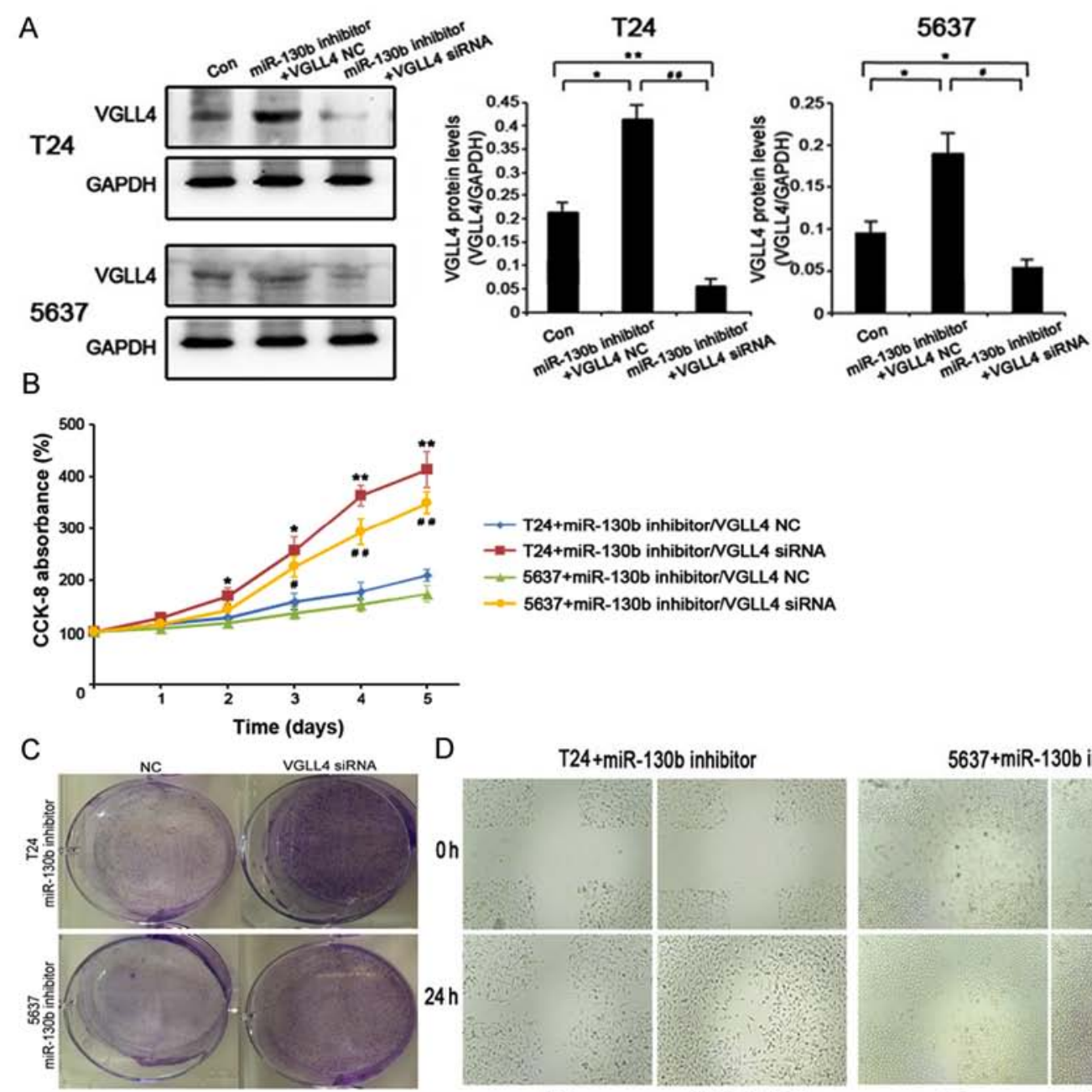

D
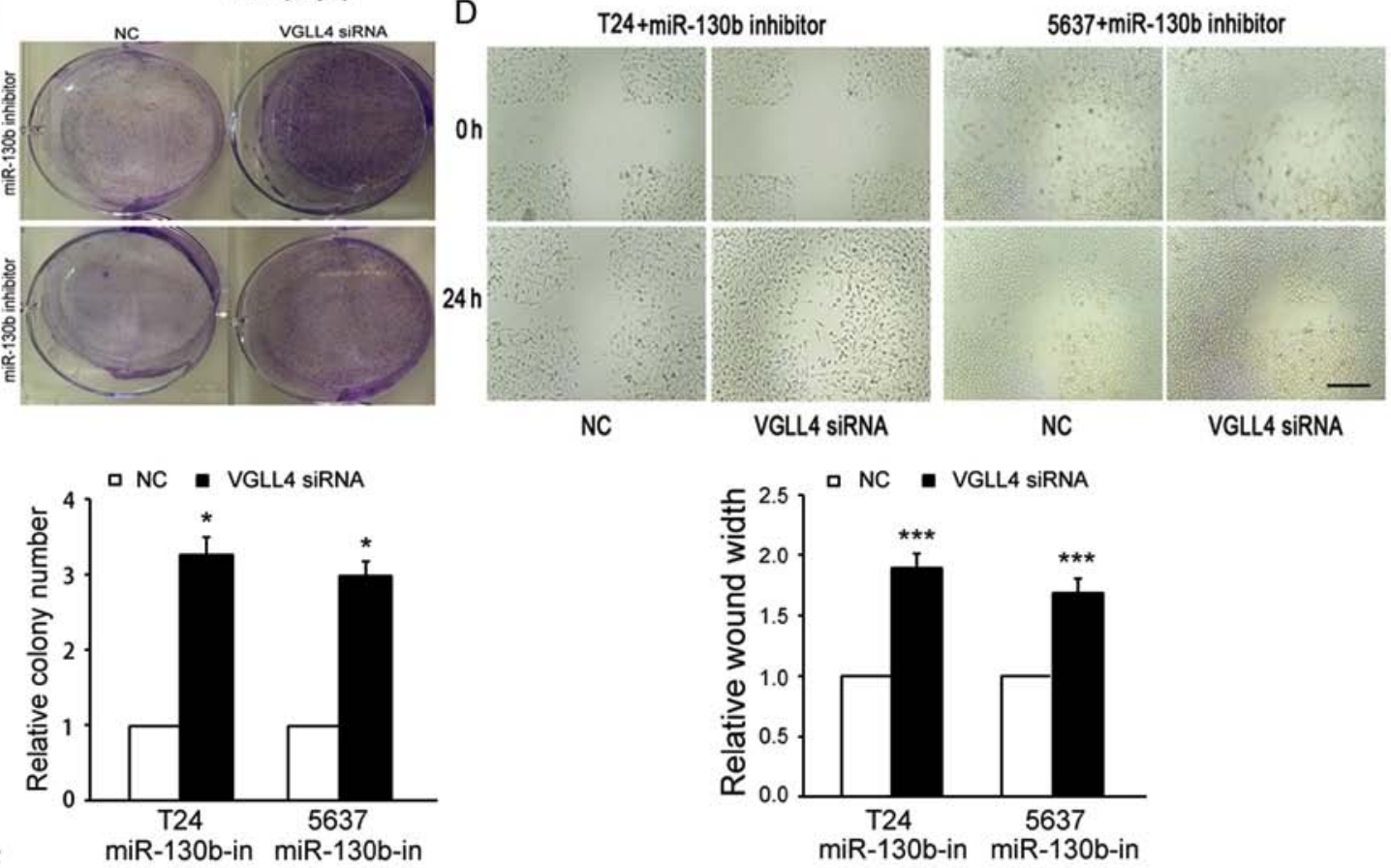

$\mathrm{E}$

NC

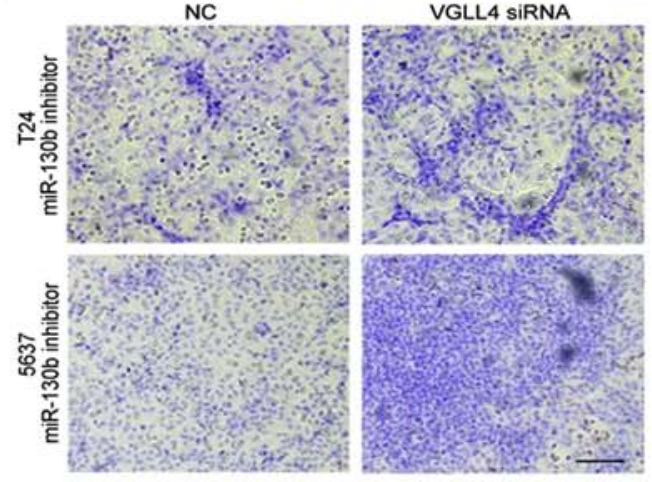

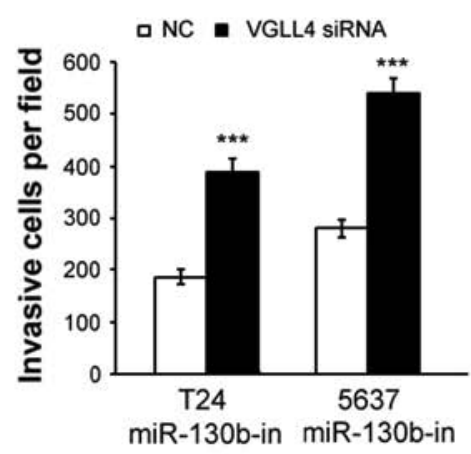

Figure 5. miR-130b promotes bladder cancer (BCa) cell proliferation, migration and invasion by inhibiting VGLL4. (A) Western blotting was performed to measure VGLL4 expression levels in miR-130b-inhibitor transfected bladder cells (T24 and 5637) transfected with VGLL4-siRNA and a control vector. (B) CCK-8 assay was performed to detect the cell proliferation ability of the indicated cells. (C) Representative micrographs and quantification of crystal violet staining in the colony formation assay of the indicated cells. (D) Effects on the migration ability of the indicated cells, as demonstrated by wound healing assay; Scale bar, $100 \mu \mathrm{m}$. (E) Representative results of the invasion assays of the indicated cells; Scale bar, $100 \mu \mathrm{m}$. Data represent the mean \pm SD of three independent experiments; ${ }^{*} \mathrm{P}<0.05,{ }^{* *} \mathrm{P}<0.01,{ }^{* * * *} \mathrm{P}<0.001 ;{ }^{*} \mathrm{P}<0.05,{ }^{\# \#} \mathrm{P}<0.01$. 
and reduced the migration of chemoresistant BCa cells (37). In the present study, VGLL4 protein expression levels in $\mathrm{BCa}$ tissues were significantly decreased compared with those in adjacent non-tumor bladder tissues, and the inhibitory effects of the miR-130b inhibitor on BCa cell proliferation, migration and invasion were significantly reversed by VGLL4 suppression. Furthermore, we found that the VGLL4 gene is a direct target of miR-130b and that miR-130b downregulated VGLL4 expression. miR-130b overexpression and knockdown in $\mathrm{BCa}$ cells decreased and increased VGLL4 protein levels, respectively, but did not significantly affect VGLL4 mRNA levels. These findings indicated that miR-130b played an important role in regulating VGLL4 translation rather than VGLL4 mRNA degradation. Therefore, what is the specific role of VGLL4 in BCa is to be further confirmed in our next studies.

In summary, our results indicate that miR-130b, a potential oncogene, decreased VGLL4 expression by directly binding to the 3'UTR of VGLL4 mRNA and promoted BCa cell proliferation, migration and invasion. Thus, miR-130b/VGLL4 may be a new target for the diagnosis and treatment of $\mathrm{BCa}$.

\section{Acknowledgements}

The language of our manuscript had been polished by the Nature Publishing Group Language Editing (NPG Language Editing).

\section{Funding}

The present study was supported by grants from the National Natural Science Foundation of China (grant no. 81372723).

\section{Availability of data and materials}

The datasets supporting the conclusions of this article and its additional files are available upon reasonable request.

\section{Authors' contributions}

The study was conceived by XL, CK and ZZ. Experiments were come into effect by XL. Statistical analysis was carried out by XL. Manuscript was written by XL. All authors recognized the final manuscript. All authors read and approved the manuscript and agree to be accountable for all aspects of the research in ensuring that the accuracy or integrity of any part of the work are appropriately investigated and resolved.

\section{Ethics approval and consent to participate}

This study was approved by the Ethics Committee of The First Hospital of China Medical University, and written informed consent was obtained from the patients in our center.

\section{Consent for publication}

Not applicable.

\section{Competing interests}

The authors declare that they have no competing interests.

\section{References}

1. Acloque H, Thiery JP and Nieto MA: The physiology and pathology of the EMT. Meeting on the epithelial-mesenchymal transition. EMBO Rep 9: 322-326, 2008.

2. Jemal A, Siegel R, Ward E, Hao Y, Xu J and Thun MJ: Cancer statistics, 2009. CA Cancer J Clin 59: 225-249, 2009.

3. Luke C, Tracey E, Stapleton A and Roder D: Exploring contrary trends in bladder cancer incidence, mortality and survival: Implications for research and cancer control. Intern Med J 40: 357-362, 2010.

4. van Rhijn BW, Burger M, Lotan Y, Solsona E, Stief CG, Sylvester RJ, Witjes JA and Zlotta AR: Recurrence and progression of disease in non-muscle-invasive bladder cancer: From epidemiology to treatment strategy. Eur Urol 56: 430-442, 2009.

5. Tan YG, Eu E, Lau Kam On W and Huang HH: Pretreatment neutrophil-to-lymphocyte ratio predicts worse survival outcomes and advanced tumor staging in patients undergoing radical cystectomy for bladder cancer. Asian J Urol 4: 239-246, 2017.

6. Urquidi V, Netherton M, Gomes-Giacoia E, Serie DJ, Eckel-Passow J, Rosser CJ and Goodison S: A microRNA biomarker panel for the non-invasive detection of bladder cancer. Oncotarget 7: 86290-86299, 2016.

7. Falzone L, Candido S, Salemi R, Basile MS, Scalisi A McCubrey JA, Torino F, Signorelli SS, Montella M and Libra M: Computational identification of microRNAs associated to both epithelial to mesenchymal transition and NGAL/MMP-9 pathways in bladder cancer. Oncotarget 7: 72758-72766, 2016.

8. Amir S and Mabjeesh NJ: microRNA expression profiles as decision-making biomarkers in the management of bladder cancer. Histol Histopathol 32: 107-119, 2017.

9. Shin SS, Park SS, Hwang B, Moon B, Kim WT, Kim WJ and Moon SK: MicroRNA-892b influences proliferation, migration and invasion of bladder cancer cells by mediating the $19^{\mathrm{ARF}} / \mathrm{cyclin} \mathrm{D} 1 / \mathrm{CDK} 6$ and Sp-1/MMP-9 pathways. Oncol Rep 36: 2313-2320, 2016.

10. Bartel DP: MicroRNAs: Genomics, biogenesis, mechanism, and function. Cell 116: 281-297, 2004.

11. Wang D, Qiu C, Zhang H, Wang J, Cui Q and Yin Y: Human microRNA oncogenes and tumor suppressors show significantly different biological patterns: From functions to targets. PLoS One 5: 5, 2010.

12. Tang J, Ahmad A and Sarkar FH: The role of microRNAs in breast cancer migration, invasion and metastasis. Int $\mathrm{J}$ Mol Sci 13: 13414-13437, 2012.

13. Chen Y, Zhang J, Wang H, Zhao J, Xu C, Du Y, Luo X, Zheng F, Liu R, Zhang H, et al: miRNA-135a promotes breast cancer cell migration and invasion by targeting HOXA10. BMC Cancer 12: $111,2012$.

14. Ochoa AE, Choi W, Su X, Siefker-Radtke A, Czerniak B, Dinney C and McConkey DJ: Specific micro-RNA expression patterns distinguish the basal and luminal subtypes of muscle-invasive bladder cancer. Oncotarget 7: 80164-80174, 2016.

15. Burmistrova OA, Goltsov AY, Abramova LI, Kaleda VG, Orlova VA and Rogaev EI: MicroRNA in schizophrenia: Genetic and expression analysis of miR-130b (22q11). Biochemistry 72 : 578-582, 2007.

16. Wu X, Weng L, Li X, Guo C, Pal SK, Jin JM, Li Y, Nelson RA, $\mathrm{Mu} \mathrm{B}$, Onami SH, et al: Identification of a 4-microRNA signature for clear cell renal cell carcinoma metastasis and prognosis. PLoS One 7: e35661, 2012.

17. Chang RM, Xu JF, Fang F, Yang H and Yang LY: MicroRNA-130b promotes proliferation and EMT-induced metastasis via PTEN/p-AKT/HIF-1 $\alpha$ signaling. Tumour Biol 37: 10609-10619, 2016.

18. Malzkorn B, Wolter M, Liesenberg F, Grzendowski M, Stühler K, Meyer HE and Reifenberger G: Identification and functional characterization of microRNAs involved in the malignant progression of gliomas. Brain Pathol 20: 539-550, 2010.

19. Yip L, Kelly L, Shuai Y, Armstrong MJ, Nikiforov YE, Carty SE and Nikiforova MN: MicroRNA signature distinguishes the degree of aggressiveness of papillary thyroid carcinoma. Ann Surg Oncol 18: 2035-2041, 2011.

20. Dong P, Karaayvaz M, Jia N, Kaneuchi M, Hamada J, Watari H, Sudo S, Ju J and Sakuragi N: Mutant p53 gain-of-function induces epithelial-mesenchymal transition through modulation of the miR-130b-ZEB1 axis. Oncogene 32: 3286-3295, 2013. 
21. Chen Q, Zhao X, Zhang H, Yuan H, Zhu M, Sun Q, Lai X, Wang Y, Huang J, Yan J, et al: MiR-130b suppresses prostate cancer metastasis through down-regulation of MMP2. Mol Carcinog 54: 1292-1300, 2015.

22. Gabriel BM, Hamilton DL, Tremblay AM and Wackerhage $\mathrm{H}$ : The Hippo signal transduction network for exercise physiologists. J Appl Physiol 1985 120: 1105-1117, 2016.

23. Jiao S, Wang H, Shi Z, Dong A, Zhang W, Song X, He F, Wang Y, Zhang Z, Wang W, et al: A peptide mimicking VGLL4 function acts as a YAP antagonist therapy against gastric cancer. Cancer Cell 25: 166-180, 2014

24. Mann KM, Ward JM, Yew CC, Kovochich A, Dawson DW, Black MA, Brett BT, Sheetz TE and Dupuy AJ; Australian Pancreatic Cancer Genome Initiative, Chang DK, Biankin AV, Waddell N, Kassahn KS, Grimmond SM, Rust AG, Adams DJ, Jenkins NA and Copeland NG: Sleeping Beauty mutagenesis reveals cooperating mutations and pathways in pancreatic adenocarcinoma. Proc Natl Acad Sci USA 109: 5934-5941, 2012.

25. Zhang W, Gao Y, Li P, Shi Z, Guo T, Li F, Han X, Feng Y, Zheng C, Wang Z, et al: VGLL4 functions as a new tumor suppressor in lung cancer by negatively regulating the YAP-TEAD transcriptional complex. Cell Res 24: 331-343, 2014.

26. Jiang W, Yao F, He J, Lv B, Fang W, Zhu W, He G, Chen J and He J: Downregulation of VGLL4 in the progression of esophageal squamous cell carcinoma. Tumour Biol 36: 1289-1297, 2015.

27. Li H, Wang Z, Zhang W, Qian K, Liao G, Xu W and Zhang S: VGLL4 inhibits EMT in part through suppressing Wnt/ $\beta$-catenin signaling pathway in gastric cancer. Med Oncol 32: 83, 2015.

28. Li N, Yu N, Wang J, Xi H, Lu W, Xu H, Deng M, Zheng G and Liu H: miR-222/VGLL4/YAP-TEAD1 regulatory loop promotes proliferation and invasion of gastric cancer cells. Am J Cancer Res 5: 1158-1168, 2015.

29. Yoshino H, Seki N, Itesako T, Chiyomaru T, Nakagawa M and Enokida H: Aberrant expression of microRNAs in bladder cancer. Nat Rev Urol 10: 396-404, 2013.
30. Bibi F, Naseer MI, Alvi SA, Yasir M, Jiman-Fatani AA, Sawan A, Abuzenadah AM, Al-Qahtani MH and Azhar EI: microRNA analysis of gastric cancer patients from Saudi Arabian population. BMC Genomics 17 (Suppl 9): S751, 2016.

31. Li M, Li J, Ding X, He M and Cheng SY: microRNA and cancer. AAPS J 12: 309-317, 2010.

32. Cheng Y, Zhang X, Li P, Yang C, Tang J, Deng X, Yang X, Tao J, Lu Q and Li P: MiR-200c promotes bladder cancer cell migration and invasion by directly targeting RECK. Onco Targets Ther 9: 5091-5099, 2016.

33. Huang B, Zhai W, Hu G, Huang C, Xie T, Zhang J and Xu Y: MicroRNA-206 acts as a tumor suppressor in bladder cancer via targeting YRDC. Am J Transl Res 8: 4705-4715, 2016.

34. Pospisilova S, Pazourkova E, Horinek A, Brisuda A, Svobodova I, Soukup V, Hrbacek J, Capoun O, Hanus T, Mares J, et al: MicroRNAs in urine supernatant as potential non-invasive markers for bladder cancer detection. Neoplasma 63: 799-808, 2016.

35. Koontz LM, Liu-Chittenden Y, Yin F, Zheng Y, Yu J, Huang B, Chen Q, Wu S and Pan D: The Hippo effector Yorkie controls normal tissue growth by antagonizing scalloped-mediated default repression. Dev Cell 25: 388-401, 2013.

36. Dong L, Lin F, Wu W, Huang W and Cai Z: Transcriptional cofactor Mask2 is required for YAP-induced cell growth and migration in bladder cancer cell. J Cancer 7: 2132-2138, 2016.

37. Ciamporcero E, Daga M, Pizzimenti S, Roetto A, Dianzani C, Compagnone A, Palmieri A, Ullio C, Cangemi L, Pili R, et al: Crosstalk between Nrf2 and YAP contributes to maintaining the antioxidant potential and chemoresistance in bladder cancer. Free Radic Biol Med 115: 447-457, 2018. 